\title{
Chemistry and Quantum Mechanics
}

\author{
J. F. Ogilvie \\ Centre for Experimental and Constructive Mathematics, Department of Mathematics, \\ Simon Fraser University, 8888 University Drive, Burnaby, British Columbia V5A 1S6 Canada, \\ Escuela de Quimica, Universidad de Costa Rica, Ciudad Universitaria Rodrigo Facio, \\ San Pedro de Montes de Oca, San Jose, Costa Rica 11501-2050, \\ Institute of Quantum Physics, Irkutsk National Research Technical University, \\ 83 Lemontov Street, Irkutsk 664074, Russian Federation
}

\begin{abstract}
Of quantum physics, quantum chemistry and quantum mechanics, the latter is least useful for both chemical education and the practice of chemistry as a science concerned with the reactions and properties of chemical substances. We show that quantum mechanics must be viewed as a collection of methods, numbering at least thirteen, that one might apply for calculations on a system of an atomic scale. Instead of quantum mechanics we advocate the quantum laws or laws of discreteness, which have simple roots in laws of conservation and which have practical applications in various areas of observations of chemical phenomena. We conclude that quantum mechanics is largely irrelevant for the general practice of chemistry.
\end{abstract}

key words quantum physics; quantum mechanics; quantum chemistry; quantum laws

\section{Introduction}

We first distinguish between quantum physics, quantum mechanics and quantum chemistry. The term quantum implies discrete -- the discreteness of a physical entity or an observable property. A first instance of a discrete nature arose in the black lines that interspersed the continuum of gradation of colour from red to violet in the spectrum of sunlight that W. Wollaston dispersed in 1802. J. J. Thomson understood cathode rays to comprise a stream of negatively charged particles; these became known as electrons that hence constituted 'quanta' of electric charge. Quantum physics thus implies an observation and analysis of phenomena having a discrete nature. Following 13 years of the 'old quantum theory' initiated by Bjerrum [1], the initial development of quantum mechanics began nearly a century ago with the work of Werner Heisenberg,. Planck had derived a formula for the spectral density of electromagnetic radiation emitted by a black body in thermal equilibrium at temperature $T$; despite the validity of the resulting formula, not only does his derivation contain errors and inconsistencies but it fails to qualify as a true quantum theory because the distribution of radiation from a black body is absolutely continuous; for that reason no quantum explanation is required, and an alternative derivation has been reported a half century ago [2]. A useful concept of a photon as a discrete carrier of electromagnetic energy arose, however, from Planck's work in that the discrete energy of a photon is proportional to its frequency, which was recognised and applied by Einstein in his 
subsequent treatment of the photoelectric effect; the latter qualifies as the first authentic quantum theory, but is separate from quantum mechanics.

\section{Methods of quantum mechanics}

Writing on "the quantum theory of molecules", Born and Heisenberg recognised in 1924 [3] that a treatment of atomic systems must be concerned with the coordinates and momenta of the constituent particles, which set the stage for Heisenberg to originate matrix mechanics the following year. One must understand clearly that quantum mechanics is neither a chemical theory, nor even a physical theory, but a collection of methods, or algorithms, applicable in calculations for systems on an atomic scale [4]. Beyond matrix mechanics twelve other methods have been developed, as summarised in table 1 .

\section{Table 1 Mathematical methods, and their originators, for calculations within quantum mechanics}

1 matrix mechanics, Heisenberg, 1925, developed with Born and Jordan

2 symbolic method, Pauli, 1926, developed by Green, 1965

3 wave mechanics, Schroedinger, 1926

4 second quantisation, Dirac, 1927

5 density-matrix formulation, von Neumann, 1927

6 variational formulation, Jordan and Klein, 1927

7 pilot-wave formulation, proposed by de Broglie 1927, developed by Bohm 1952

8 relativistic wave mechanics, Dirac, 1928

9 phase-space formulation, Wigner, 1932

10 octonionic quantum mechanics, Jordan, 1933

11 quaternionic quantum mechanics, Birkhoff and von Neumann, 1936

12 path-integral formulation, Feynman, 1948, after Wiener 1926, Dirac 1933

13 Hamilton-Jacobi formulation, Leacock and Padgett, 1983

One must recognise as important a distinction between a theory, such as Einstein's analysis of the photoelectric effect, and a mere method according to which calculations might be undertaken, such as any of those in table 1, despite the prospective power of that method. Of thirteen methods in the table, among other attributes of a particular method most include a provision for non-commutative multiplication, i.e. $a b \neq b a$. In contrast with quantum physics or quantum mechanics, quantum chemistry generally implies a practice of extensive numerical calculations with standard programs based loosely on wave mechanics. 


\section{Quantum laws}

After a consideration of chemical thermodynamics and the kinetics of chemical reactions during a study of physical chemistry according to standard texts, quantum mechanics generally follows, in the form of a discussion of first some principles and then a supposed relation to atomic structure. Although the first topic traditionally treated is the radiation from a black body, which belongs to quantum physics, this radiation lacks a discrete aspect and can hence be neglected. Einstein's contributions to the explanation of the photoelectric effect, whereby an electron is emitted from a solid substance on absorption of a photon of energy greater than a threshold that serves the role of an ionisation energy, must be regarded as the first true quantum analysis, followed by his theory of the specific heats of solid substances and the postulate of a residual energy, which Mulliken proved experimentally in 1924 [5]. As the black lines in Wollaston's spectrum are due to absorption transitions of mostly atomic species in the solar photosphere, even though molecular entities also contribute to the observed features, atomic structure is naturally a subsequent topic. Bohr's inadequate quantum theory included, as an afterthought, an explanation of the spectral lines in the Balmer series as being due to transitions of the hydrogen atom between its states of discrete energy, consistent with the analyses of Conway, Ritz and Nicholson. Bohr derived a formula to describe the angular momentum of an electron in a circular orbit; such an orbit is not an observable quantity -- only the density of electronic charge in the vicinity of an atomic nucleus is a measurable quantity. Heisenberg's principle of indeterminacy, which limits an extent of precision of simultaneous measurements on an atomic scale, is applicable to conjugate variables in three couples -- momentum and position, energy and time, and angular momentum and angle; in all three cases the products of the variables in each couple have the dimensions of the Dirac constant, $\hbar$, which is Planck's constant $h$ divided by $2 \pi$; the products have a minimum magnitude $1 / 2 \hbar$ in terms of that quantity. The true energy states of a hydrogen atom are precisely represented with four quantum numbers -- $n, l, j$, $m_{j}$, which emanated from Darwin's solution [6] of Dirac's equation according to relativistic wave mechanics. A common practice of describing states and spectra of atoms comprising two or more electrons with quantum numbers $n, l, m$ derived from Schroedinger's imperfect solution -as it is relativistically incorrect and not invariant under a Lorentz transformation -- for a hydrogen atom according to the original wave mechanics [7] lacks a scientific basis; there is hence no legitimate justification for such a description of atomic spectra as being founded on quantum mechanics. Atomic spectra are important in chemistry in relation to elemental analysis through atomic absorption, developed by Walsh after 1950, but a simplistic description of the transitions involved in these spectra in terms of quantum numbers for a hydrogen atom is clearly an extrapolation from a point and a logical error.

Some early ideas about a chemical bond, which defies an exact definition even though each chemist from an early age comprehends its practical significance, involved the notion of an electron pair. A prototypical instance of such a chemical bond is molecular dihydrogen, ${ }^{1} \mathrm{H}_{2}$; as the density of electronic charge, at the equilibrium separation between the two protons as atomic nuclei, was calculated accurately [8] at the midpoint to be less than one tenth greater than the 
corresponding charge density at that position between two hypothetically non-interacting hydrogen atoms at the same distance, a description as a 'two-electron bond' is clearly an exaggeration, despite the stability of that bond with respect to dissociation into two hydrogen atoms that requires $\sim 4.478 \mathrm{eV}$ [9]. For dilithium, which one might expect to possess also a 'twoelectron bond', the dissociation energy of $\mathrm{Li}_{2}, 1.026 \pm 0.006 \mathrm{eV}$ [10], is markedly less than that of cation $\mathrm{Li}_{2}{ }^{+}, 1.297 \pm 0.001 \mathrm{eV}$ [11], which one might likewise expect to possess a one-electron bond, as in prototypical ${ }^{1} \mathrm{H}_{2}{ }^{+}$with dissociation energy $\sim 2.65 \mathrm{eV}$ [9]. For methane various spectrometric and computational evidence might favour -- but far from definitively -- that six electrons are involved in forming four chemical bonds between $\mathrm{C}$ and $\mathrm{H}$, rather than eight electrons. For cyclopropane, concepts to rationalise the structure of this stable molecule in terms of common generalisations, such as hybridisation, are lacking. Instead of proceeding to portray the existence or stability of molecules as resulting from approximate theoretical or computational treatments or schemes, one should associate the structure and properties of a molecule with the results of experiments, such as measurements of diffraction data and spectra, that have enabled an accurate evaluation of those features to define the identity of a chemical compound or material. In essentially all diffraction experiments the sample under test exists in some set of quantum states occupied at the temperature of the experiments; the resulting structures hence reflect that same variety of states, rather than a particular state specified with pertinent quantum numbers in an appropriate set applicable to a spectrometric experiment.

In such a spectrometric experiment, whether on a macroscopic sample or a molecular beam, an analysis of the observations is best based on the quantum laws [12] of molecules and photons, as outlined in table 2. An interpretation or treatment of any significant experiment is impracticable without a model or principles as a basis and a knowledge of pertinent properties of the samples upon which the measurements are made. For an interpretation of spectra, we adopt a position that is independent of any theory other than basic classical mechanics and of electric and magnetic fields; we thus avoid special theories, in particular those known as quantum theories, that have been developed to rationalize ex post facto the features of spectra of interest. We recognise instead, and apply, the quantum laws, or the laws of discreteness, which we describe as follows.

Table 2 Quantum laws or laws of discreteness of molecules and photons

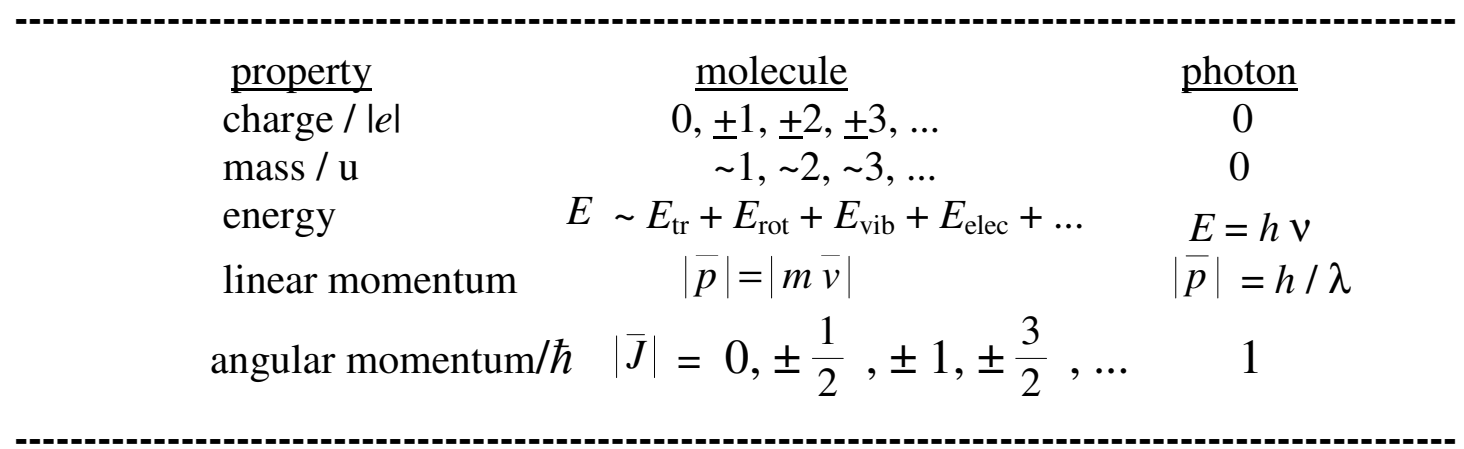


We consider here that the universe comprises a sum of matter and radiation; that these constituents are interconvertible is immaterial for practical chemistry -- the preparation and analysis of chemical substances and their mixtures. Matter might be stationary or in motion, relative to an observer, but radiation is always in motion: even though there are cavities that contain radiation, for instance as standing waves of microwave radiation within a reflex klystron or in a laser cavity, we consider that radiation to be in a continuous motion without frictional loss, reflected between mirrors or from one side of a cavity to another. Experiments have been designed and performed to take advantage of the duration of that transit back and forth across a cavity: so-called cavity ring-down spectra yield useful results for transitions that are otherwise too weak for convenient measurement. Both matter and radiation have their electric and magnetic aspects: with radiation, visible light for instance, is associated an electromagnetic field, which exists even in the absence of matter; radiation from our sun, whether as radio waves or visible light or xrays, thus reaches the terrestrial environment through the intervening vacuum i.e. the complete absence of matter, that can still support an electromagnetic field. Likewise, macroscopic matter, within which the atomic centres of the formula units comprise positively charged atomic nuclei and the associated negatively charged electrons, can bear a net electric charge, such as a plate of an electric capacitor, or a magnetic dipolar moment, such as a bar of iron or iron oxide; matter in a form of a microscopic entity such as a molecule can analogously bear a net electric charge or a net electric or magnetic dipolar moment.

Although, on a conventional or macroscopic scale, both matter and radiation seem continuous and to be capable of subdivision or attenuation ad libitum, on a microscopic or atomic scale both matter and radiation are found experimentally to comprise discrete units; such a discrete unit might be called a quantum, or described as the result of its discrete nature. The word quantum is adapted from latin quantus, meaning how much; in English, a quantum signifies a chunk. The discrete unit of matter we call a molecule -- an isolated atom is merely a molecule with a single atomic centre; decomposition of an atom into its constituent atomic nucleus and separate electrons yields an unstable system of little chemical interest. The discrete unit of radiation we call a photon, a term proposed by G. N. Lewis in 1926 to signify a carrier of radiant energy. Einstein's interpretation of the photoelectric effect in 1905 was the first substantive recognition of the discreteness of radiation; that photoelectric effect is applied in vacuum phototubes or photomultiplier tubes that serve as sensitive detectors in spectrometers for the near-infrared, visible and ultraviolet regions. Chemists almost never work with either a single molecule or a single photon, but only ensembles of these entities of sufficiently large number. For most practical purposes, we can consider a (small) molecule to have dimensions typically of order $10^{-9} \mathrm{~m}$ or $1 \mathrm{~nm}$, but in principle a piece of matter as large as a diamond claimed to be the size of a coconut, or a single crystal of ordinary salt, $\mathrm{NaCl}$, as large as a football, or a wide sheet of polyethene, might constitute a single molecule according to conventional chemical criteria. Likewise, for most practical purposes, we can ignore any internal structure of a photon, but, in a chemical context, a molecule is usefully considered to be composed of atomic nuclei and their surrounding electrons. According to conventional definitions, an atomic nucleus in a stable or enduring molecular system bears a net positive electric charge, whereas each associated external electron bears a negative charge; enduring molecules exhibit electric neutrality such that the total number of protons, or a sum of the atomic numbers, equals the number of constituent electrons. In the gaseous phase some cations such as $\mathrm{NO}^{+}$or anions such as $\mathrm{CN}^{-}$can have a transient existence in experiments during which their properties become measurable. Matter comprises materials, in condensed or dense phases, and molecules. As a separate entity, a molecule exists rigorously only in an isolated state, such as in an hypothetical ideal gas or as a constituent of an 
interstellar cloud, having number density $\sim 5$ molecules $\mathrm{mL}^{-1}$, and in an absence of external fields -- which formally never occurs; to an approximation of extent variable, depending on both the chemical nature of a sample and the nature of an experimental measurement on that sample, a (small) molecule might, however, seem to exist in a recognizable manner not just in a dilute gaseous sample but also within a condensed phase - solid, liquid or mesophase. Within a molecule, there exist only atomic nuclei and electrons - hence no atoms, but, near an atomic nucleus an atomic centre is observable that might resemble an isolated atom of the same atomic number, except the case of hydrogen. The discrete properties of a molecule or a photon number at least five according to the table above: four of them, which conform to macroscopic descriptions, are inertial mass, electric charge, total energy and linear momentum. A further property has the dimensions of a physical quantity called action, or angular momentum, or possibly something else; this property is known generally as angular momentum, which implies a rotation of some object, but the nature of what might be rotating on a microscopic scale is uncertain in some cases, such as an electron or proton. Mass, charge and energy are scalar quantities, but linear momentum and angular momentum (or whatever other quantity might pertain) are vectorial quantities.

These properties are all directly observable from experiment; for instance, not only the discreteness of electric charge but also a value of electronic charge, at present defined as $e=$ $-1.60217663410^{-19} \mathrm{C}$, were determined in Millikan's experiment with drops of oil. With each property is associated a law of conservation -- hence conservation of net electric charge, of mass, of energy, of linear and angular momentum; although mass and energy must formally be treated in combination, for chemical purposes each might be accounted separately. These laws are applicable on a chemical or atomic scale, not a sub-nuclear scale. Each conserved quantity -mass and energy, electric charge, linear and angular momentum -- is supposed to reflect some elementary symmetry, but for an interpretation of spectra or phenomena and other properties of chemical systems such aspects are generally irrelevant.

In a spectrometric experiment, one measures the wave length, $\lambda$, or frequency, $v$, of the electromagnetic waves in the form of photons, or an equivalent property such as wavenumber $\bar{v}$ $=\mathrm{v} / \mathrm{c}$, with speed $c$ of electromagnetic wave that represents the magnitude of the phase velocity; in either case, such a measurement implies an energy, $E=h \mathrm{v}=h c / \lambda$ of the associated photon as a difference of energies between two states of some matter or molecule under test. For absorption or emission by a molecule or material, the absorbed or emitted photon represents an energy difference $\Delta E$ between the energies of two states of that absorber or emitter, so $\Delta E_{\text {abs }}$ or $\Delta E_{\mathrm{em}}=E_{\text {photon }}$, but that measurement conveys no information about possible frequencies of the absorber or emitter, only a difference of energy. In 1920 Kemble recognised that the frequency of a transition in a spectrum implies the frequency of the electromagnetic wave that interacts with an atom or molecule; that frequency is quite separate from, and unrelated to, any purported frequency of a motion of, or within, an atom or molecule [13]. In a scattering process, the difference of energy between the energy of an incident photon and the energy of the scattered photon is equal to a difference of energy of the scatterer, which might be a decreased energy in a Stokes process or an increased energy in an anti-Stokes process. For measurements of a wave length that involve a passage of the detected light through air or other medium, a correction for the index of refraction is appropriate to yield an accurate frequency or energy.

We consider each property in turn. A photon bears neither rest mass nor charge, but a (small) molecule of particular isotopic composition bears a rest mass or inertial mass that is nearly an integer in terms of unified atomic mass unit, $\mathrm{u}$, or dalton, Da; a large crystal of salt or that enormous diamond might have a mass of order kilogram, which corresponds to $\sim 10^{27} \mathrm{u}$ and 
for which any relation to an integer is meaningless. A molecular entity can bear a net electric charge, of value 0 for a net neutral molecule, or $\pm 1, \pm 2, \ldots$ for a molecular ion such as $\mathrm{NO}^{+}$or $\mathrm{OH}^{-}$or $\mathrm{Ca}^{2+}$, in units of charge $e$ on a proton, which is the same magnitude as the charge on a single electron but of opposite sign. The intrinsic energy of a photon is directly proportional to the frequency, $v$, of an associated electromagnetic wave; the factor $h$ of proportionality is known as Planck's constant, of defined value $h=6.6260701510^{-34} \mathrm{~J}$ s. A free molecule might exist in a state of discrete energy, although a continuum of internal energy is also possible under various conditions, such as above a dissociation limit or an ionisation limit; above that limit the individual fragments might have discrete energies, but the ensemble has continuous energy including the relative kinetic energy. Apart from the intrinsic mass energy and the internal states of atomic nuclei, that molecular energy is conventionally subdivided approximately into classical contributions of translation, or external kinetic energy, rotation of the molecule as a whole about an internal axis, internal vibrations of atomic centres, and electronic energy; each such contribution to the total energy might also assume discrete values, and vibrational and electronic energy might be considered to have kinetic and potential contributions.

A photon moves in vacuo at speed $c$ independent of its frequency; its linear momentum is inversely proportional to wave length $\lambda$ of the associated electromagnetic wave; the factor of proportionality is again Planck's constant, so $|\bar{p}|=h / \lambda$. For a molecule confined to an enclosed space, its linear momentum, taken as a product of its mass and velocity, seems to assume discrete values, but the linear momentum of a free molecule, for instance in an interstellar cloud, assumes continuous values. A photon has unit angular momentum in terms of the Dirac constant, independent of frequency or wave length; a photon is thus rigorously a boson, or particle to which Bose-Einstein statistics pertain. A single molecule has total angular momentum in integer or half-integer multiples of $\hbar$; including the effects of intrinsic nuclear angular momentum, a molecule is thus either a boson if it possess zero or integer total angular momentum in terms of $\hbar$, such as free ${ }^{12} \mathrm{C}^{16} \mathrm{O}$ in its electronic state of least energy, or a fermion, to which Fermi-Dirac statistics pertain, if it possess $\frac{1}{2}, \frac{3}{2}, \frac{5}{2}, \ldots$ units of angular momentum in terms of $\hbar$, such as ${ }^{3} \mathrm{He}$ or ${ }^{2} \mathrm{H}$. The external kinetic energy of a molecule is related to its linear momentum, which affects only indirectly the internal energy of which one might measure a change during absorption or emission or scattering of a photon. The angular momentum of a molecule is, in contrast, of great importance in relation to radiative processes; not only the total angular momentum as a vectorial quantity takes discrete values, such as $0,1,2, \ldots$, but also a component of angular momentum along a particular axis, generally named axis $z$, takes discrete values of interval 1 unit. For instance, for total angular momentum 2 units, in terms of $\hbar$, the component takes values from -2 to 2 , so $-2,-1,0,1,2$ in the same unit. The total angular momentum of a photon is 1 unit, and its components comprise only -1 and +1 , not 0 ; these values might be roughly associated with circularly polarized light, with rotation clockwise or anticlockwise, for instance. Like energy, angular momentum can, in the absence of friction, be neither created nor destroyed, but might be exchanged between particles or between a particle and a radiation field; this conservation of angular momentum is considered to be a direct consequence of the isotropy of space, i.e. the fact that empty space has the same properties in all directions.

These discrete properties imply the existence of quantum numbers, independent of any quantum theories. For a net electric charge, the possible values for a molecular entity in units of $e$ are positive and negative integers, $0, \pm 1, \pm 2, \pm 3, \ldots$. For mass, the possible values for neutral molecules are positive integers $1,2,3, \ldots$ as mass numbers, but these values are only approximately integers in terms of unified atomic mass unit or dalton; for two molecules of the 
same total mass number, such as ${ }^{12} \mathrm{C}^{16} \mathrm{O}$ and ${ }^{14} \mathrm{~N}_{2}$ each of mass number 28 , there are slightly disparate actual masses, $27.99491461956 \mathrm{u}$ for ${ }^{12} \mathrm{C}^{16} \mathrm{O}$ and $28.00614800956 \mathrm{u}$ for ${ }^{14} \mathrm{~N}_{2}$, for instance, that produce separate lines in a mass spectrum of a mixture of these two compounds at sufficiently great resolution. As the mass, $(5.48579909065 \pm 0.00000000016) \times 10^{-3} \mathrm{u}$, of an electron is much smaller than the mass of any stable atomic nucleus, such as a proton of mass $(1.007276466621 \pm 0.000000000053) \mathrm{u}$, the mass of a molecular ion accordingly differs only slightly from that of the corresponding neutral atom or molecule. For the energy of an isolated diatomic molecule with hence two atomic centres, the vibrational contributions to the total energy might be described in terms of integer $0,1,2,3$, .., but the energy deviates markedly from a linear relation beyond small values of this quantum number. The energy of overall molecular rotation is formally only approximately separable from energy due to angular momenta in other forms, including intrinsic electronic and nuclear angular momenta, or from vibration; the energy of electronic excitation of atoms or molecules is generally not expressible in terms of simple relations involving integers; atomic $\mathrm{H}$ or other one-electron atom is an exception in this regard. A quantum number for linear momentum is generally of no practical interest, whereas a quantum number for angular momentum of an atom or molecule, which assumes integer and half-integer values in terms of Dirac constant $\hbar$, commands the utmost importance: the internal state of a molecule, hence excluding its external kinetic energy, might be defined uniquely in terms of its total internal energy and its total angular momentum expressed in terms of quantum numbers.

These attributes constitute the quantum laws of nature, or, more precisely, the laws of discreteness. The separate spectral lines, as distinct from a continuum or continuous spectrum, that we can observe for various samples under appropriate diverse experimental conditions, such as optical spectra of gaseous constituents and mass spectra of ions, constitute the first and strongest evidence for these laws of discreteness.

\section{Application of quantum laws}

A student of chemistry learns early how to balance a chemical equation, so to evaluate an stoichiometric coefficient of each reactant and product species involved in a particular reaction as an integer, without explicit recourse to quantum laws, even though the conservation of mass and charge are directly involved in this process. When the level of a chemical equation increases to take into account the enthalpies of formation of the various species, yielding an enthalpy change for the reaction, the conservation of energy is effectively included but with no particular discrete aspect. The quantum laws really become most directly observable in the analysis of absorption or emission spectra of small molecules.

In physical chemistry, after a superficial view of atomic spectra the rotational spectra of diatomic molecules are customarily introduced. Such an absorption spectrum of a substance such as gaseous $\mathrm{CO}$ or $\mathrm{HCl}$ under ambient conditions comprises a sequence of nearly equidistant lines in the far-infrared region. Such discrete lines are undeniable evidence of the discreteness of some molecular property. If a sample could be measured at a temperature near $0 \mathrm{~K}$, which is nearly practicable with a jet employing adiabatic expansion, only one line would appear. Under such conditions practically all molecules are in the ground state, characterised with quantum number $J=0$. Because about 30 lines are readily observable for a gaseous sample near $300 \mathrm{~K}$, 
there are clearly many states of the molecule that could be occupied as a result of a transition from that ground state, but only one transition appears. A simple explanation relies on the fact that a photon has one unit of angular momentum, in terms of $\hbar$; on absorption of a photon the molecule hence gains one unit of angular momentum, and only that transition is possible that involves such a gain of one unit. As the temperature of the gaseous sample increases, further states become thermally occupied, from which transitions on absorption of photons with appropriate energies can occur with $\Delta J=+1$, such that there is adherence to the laws of conservation of both energy and angular momentum. The fact that there is only one line observable for $\mathrm{CO}$ at the lowest temperatures also implies that angular momentum is acquired by a molecule in gaining rotational energy, because there is angular momentum of no other type applicable for ${ }^{12} \mathrm{C}^{16} \mathrm{O}$ that has no unpaired electron and no intrinsic nuclear angular momentum. Each rotational state is characterised with a parity; this parity is either even for states with $J$ equal to even numbers, $J=0,2,4, .$. , or odd otherwise; transitions in absorption or emission occur only between states of opposite polarity. In a rotational Raman spectrum of a simple diatomic molecule, transitions occur with $\Delta J=+2$, because two photons are involved in a scattering process -- one incident and the other scattered; there is thus no change of parity between initial and final states of a transition.

For a gaseous sample containing diatomic molecules at a temperature not more than about $300 \mathrm{~K}$, absorption spectra in the mid- and near-infrared region under poor resolution reveal a sequence of features of rapidly decreasing intensity as frequency or wavenumber increases. The relative insensitivity of the presence of these coarse features to temperature indicates that angular momentum is not directly involved in their nature. As rotational motion is already taken into account related to the spectrum in the far-infrared region, these features are assignable to vibrational motion, which is consistent with the isotope effect for $\mathrm{HCl}$ and $\mathrm{DCl}$. Upon improved resolution for a sample such as $\mathrm{CO}$ or $\mathrm{HCl}$, each feature proves to be composite, comprising two sequences of lines (or two double sequences for $\mathrm{HCl}$ because of the natural abundance of ${ }^{1} \mathrm{H}^{35} \mathrm{Cl}$ and ${ }^{1} \mathrm{H}^{37} \mathrm{Cl}$ ), with a gap between these sequences. At low temperatures, $T<10 \mathrm{~K}$, only one line is observed [14] experimentally for $\mathrm{DCl}$; such a phenomenon is again symptomatic of the involvement of angular momentum, as for the pure rotational spectrum in absorption explained above. (Although these effects of temperature that require adiabatic expansion from a supersonic jet for direct observation are difficult to create in an ordinary laboratory for physical chemistry, one can readily make animated simulations of these effects to illustrate the primary features.) As the temperature of the sample is increased somewhat from $12 \mathrm{~K}$, two further lines appear; on further increase these features become two sequences of lines, each called a branch. The branch at wavenumber greater than that of the central gap is named R; its lines are readily explicable as involving an increase of rotational quantum number $J$ between the ground vibrational state and a state of increased vibrational energy, but with $\Delta J=+1$ as a molecule absorbs a photon and increases both its energy and angular momentum. For the other branch, named $\mathrm{P}$, that proceeds from the central gap to smaller wavenumber, the corresponding condition is a gain of energy but a loss of angular momentum by one unit, i.e. $\Delta J=-1$; this condition occurs because angular momentum is a vectorial quantity, so that a 'sum' of vectors can become effectively a difference. 
In the Raman scattering spectrum of the vibration-rotational band three branches appear, one with closely spaced lines at the gap in the absorption spectrum, and the other two, having intervals roughly twice the interval in each branch of the absorption spectrum, spreading out from that central branch, named Q. These branches correspond to two units of angular momentum added to, or subtracted from, -- in a vectorial sense -- the value of $J$ before the scattering event, so $\Delta J=+2$ for branch $S$ to greater wavenumber, $\Delta J=-2$ for branch $\mathrm{O}$ to smaller wavenumber, with $\Delta J=0$ for branch $\mathrm{Q}$ at the centre of the band, for which the angular momentum of one photon cancels the angular momentum of the other photon in the scattering process. Each such transition due to Raman scattering occurs between states of the same parity.

For the pure rotational spectrum of the diatomic molecule in absorption, the single branch is also named $\mathrm{R}$, whereas for the pure rotational spectrum in Raman scattering, which is observable on either side of the exciting line with twice the intervals in the absorption spectrum, both branches are named S, consistent with the vibration-rotational Raman spectra as treated above. If the exciting line does not excessively overwhelm lines closely spaced between the two $\mathrm{S}$ branches, branch $\mathrm{Q}$ might be discernible beside that exciting line.

For triatomic molecules having a linear conformation of the atomic centres at equilibrium a further readily observable effect involving angular momentum arises. The bands associated of a gaseous sample involving vibrational motions parallel to the axis of the equilibrium linear conformation, described as bond-stretching modes, show only two rotational branches in absorption, so $\mathrm{P}$ and $\mathrm{R}$ on either side of a gap at the centre that marks the pure vibrational transition, just as for a diatomic molecule such as $\mathrm{CO}$ or $\mathrm{HCl}$; that pure vibrational transition is not observable in an absorption spectrum because an absorbed photon would not have its angular momentum conserved with $\Delta J=0$. When the molecular geometry of a triatomic molecule with a linear equilibrium conformation, such as $\mathrm{HCN}$ or $\mathrm{N}_{2} \mathrm{O}$, becomes non-linear as a result of an angular deformation, that deformation is related to the same deformation with the opposite phase in two ways -- either through that angular deformation or as a result of a rotation of the molecule about the axis corresponding to the collinear conformation; this effect is hence tantamount to a vibrational angular momentum. This angular deformation is, furthermore, doubly degenerate, such that, with the equilibrium collinear nuclear conformation along axis $z$, an angular deformation can occur in two mutually perpendicular planes, such as plane $x z$ and $y z$; a further quantum number $l$ for this vibrational angular momentum hence arises. For a vibrational state in which the molecule retains its linear equilibrium conformation, the value of quantum number $l$ is 0 . For the ground vibrational state of a molecule such as $\mathrm{HCN}$ or $\mathrm{N}_{2} \mathrm{O}$, there is hence no vibrational angular momentum and $l=0$. Each rotational state within an excited vibrational state associated with an angular deformation to the extent of one unit, so with $l= \pm 1$, becomes doubled through the specified degeneracy of the vibrational mode, with one state of even parity and the other of odd parity; for a given value of $J$ there is a tiny separation of energy between the two components. The rotational structure associated with a vibration of type angular deformation thus comprises not only perceptible branches $\mathrm{P}$ and $\mathrm{R}$ but also a central branch $\mathrm{Q}$; from a particular rotational state with one parity in the vibrational ground state the transitions with $\Delta J=$ \pm 1 occur to one component of rotational state $J$, with the opposite parity, in the vibrationally 
excited state; transitions with $\Delta J=0$ occur to the other component with the other parity. This description is slightly simplified but it suffices to indicate roughly how a vibration-rotational band associated with an angular deformation of a triatomic linear molecule exhibits branch Q as well as branches $\mathrm{P}$ and $\mathrm{R}$, in each case maintaining a conservation of angular momentum. Analogous explanations are applicable to spectra of other gaseous samples containing tetratomic and larger linear molecules.

Although these applications of the quantum laws provide quantitative accounts of the frequency aspects of spectral features commonly met in spectra of simple gaseous chemical compounds or discussed in physical chemistry, they are inadequate for the intensity aspects. Even most qualitative features of intensities require significant calculations, according to one or other quantum-mechanical method, that are simply omitted in practice, relying instead on statements about matrix elements or expectation values that are simply proffered without a justifying calculation. These intensities are just as important in a total quantitative treatment of molecular spectra as the frequencies: according to Heisenberg, the observable features of a (free) atom or molecule are the frequencies and intensities of their spectral lines [15]. A direct treatment of the frequency aspect nevertheless provides a satisfactory initial account of optical spectra; instead of comparison with the results from calculations involving a mythical rigid rotor or harmonic oscillator, the quantum laws provide a valid and useful explanation on a basis of simple physical principles readily comprehended as an extension of the laws of conservation to become the laws of discreteness.

\section{Conclusion}

What we seek to explain in this essay is that quantum mechanics is generally irrelevant for the practice of chemistry, apart from the fact that chemists generally lack the mathematical knowledge and expertise to appreciate the fabric of quantum-mechanical methods. The most important single aspect of chemistry is the structure of molecules and materials, in terms of which the reactions and properties of chemical substances become characterised and understood. Spectroscopy plays a major role in the practice of analytical, inorganic and organic chemistry in the form of infrared, ultraviolet, mass and especially nuclear-magnetic-resonance spectra that serve to identify and to characterise the products of chemical synthesis and degradation, with atomic-absorption spectra for the analysis of chemical elements, to clarify and to define the structures of chemical substances and materials. All these spectra with discrete features involve the quantum laws. Because molecular structure is incompatible with quantum mechanics, in principle [16] and in practice [17], for what reason should chemists bother to become acquainted with quantum mechanics? Orbitals and all associated nonsense that are mere artefacts of one particular method of quantum mechanics are absolutely redundant. As a collection of methods for calculations applicable to systems on an atomic scale, quantum mechanics is a legitimate component of a study of physics, like relativity theory; in both cases their direct involvement in chemical operations and the analysis of chemical data is of minuscule importance for all practical purposes. Quantum physics that is concerned with the observation and analysis of experiments that exhibit discrete features has relevance to chemistry greater than quantum mechanics because such phenomena as the photoelectric effect and the existence of line spectra in regions from the microwave region to the hard xray region, including every region between, have practical applications in the operation of instruments in chemical laboratories and in the chemical analysis

of matter in all states of aggregation. The most valuable knowledge for these applications 
constitutes the quantum laws or laws of discreteness, particularly the related conservations of both energy and angular momentum, as discussed above. Quantum-chemical calculations also are useful for chemical purposes, but the nature of present computer programs, even those freely available, facilitates the computations of properties of chemical interest, such as molecular structure in the form of bond lengths and bond angles, or electric dipolar moments, but require little or no knowledge about any particular method of quantum mechanics for their successful operation; the proof of this assertion is that these programs are occasionally used, generally injudiciously, in courses in chemistry before the principles and dogma of quantum mechanics are administered to those students. These quantum-chemical calculations must be regarded as semiempirical: they are far from being "from first principles" or ab initio because a tentative structure must be input before a calculation begins and because the basis sets are canned -- calibrated with experimental data. For many structures of chemical interest, alternative programs described as molecular mechanics [18] to conduct calculations, based on readily understood simple physical principles and designed to operate with parameters of empirical origin, yield similar information of generally sufficient accuracy with much decreased duration and no direct connection with quantum mechanics.

\section{STATEMENT REGARDING CONFLICT OF INTEREST}

The author states no conflict of interest. 


\section{References}

1 J. F. Ogilvie, The first quantum theory of molecules, Resonance, 19, 834-839, 2014

2 T. H. Boyer, Classical statistical thermodynamics and electromagnetic zero-point radiation, Physical Review, 186, 1304-1318, 1969

3 M. Born and W. Heisenberg, The quantum theory of molecules, Annelen der Physik, 74, $1-31,1924$

4 P. Ball, https://www.bbc.com/future/article/20130124-will-we-ever-get-quantum-theory

$5 \quad$ R. S. Mulliken, The band spectrum of boron monoxide, Nature, 114, 349-350, 1924

6 C. G. Darwin, The wave equations of the electron, Proceedings of the Royal Society, London, 117, 118, 654-680, 1928

7 E. R. J. A. Schroedinger, Quantisation as a problem of proper values I, Annelen der Physik, 79, 361-376, 1926, translated into English in Collected Papers on Wave Mechanics, AMS Chelsea Publishing, Providence RI USA, third edition, 1982

8 R. Moszynski, S. Krzysztof, Accurate electron densities of the hydrogen molecule, Journal of Physics B, Atomic and Molecular Physics, 20, 4347-4364, 1987

9 NIST chemistry webbook, https://webbook.nist.gov/chemistry/

10 R. Velasco, C. Ottinger, R. N. Zare, Dissociation energy of $\mathrm{Li}_{2}$ from laser-excited fluorescence, Journal of Chemical Physics. 51, 5522-5532, 1969

11 S. Magnier, S. Rousseau, A.R. Allouche, G. Hadinger, M. Aubert-Frecon, Potentialenergy curves of 58 states of $\mathrm{Li}_{2}{ }^{+}$, Chemical Physics 246, 57-64, 1999

12 J. F. Ogilvie, The nature of the chemical bond 1990 -- there is no such thing as orbital, Journal of Chemical Education, 67, 280-289, 1990

13 E. C. Kemble, The Bohr theory and the approximate harmonics in the infrared spectra of diatomic gases, Physical Review, 15, 95-109, 1920

14 S. Klee, J. F. Ogilvie, The fundamental vibration-rotational band of gaseous DCl in absorption at $297 \mathrm{~K}$ and at $12 \mathrm{~K}$, Spectrochimica Acta, 49A, 345-355, 1993

15 W. Heisenberg, On an alteration to the formal rules of quantum theory in a problem of anomalous Zeeman effects, Zeitschrift fur Physik, 26, 291-307, 1924

16 R. G. Woolley, Quantum theory and molecular structure, Advances in Physics, 25, 27-52, 1976

17 M. Cafiero, L. Adamowicz, Molecular structure in non-Born-Oppenheimer quantum mechanics, Chemical Physics Letters, 387, 136-141, 2004

18 E. G. Lewars, Computational Chemistry, edition 3, 2016, Springer, Switzerland 